\title{
Magnetic Doping and Characterization of n-type GaN
}

\author{
X.M. Cai ${ }^{\mathrm{a}}$, A.B. Djurišićc ${ }^{\mathrm{a}}$, M.H. Xie ${ }^{\mathrm{a}}$, H. Liu ${ }^{\mathrm{b}}$, X.X. Zhang ${ }^{\mathrm{b}}$, J.J. Zhu ${ }^{\mathrm{c}}$, H. Yang ${ }^{\mathrm{c}}$, \\ Y. H. Leung ${ }^{\mathrm{a}}$ \\ ${ }^{a}$ Dept. of Physics, the University of HongKong, HongKong, China \\ ${ }^{b}$ Dept. of Physics, the Hong Kong University of Science and Technology, HongKong, China \\ ${ }^{c}$ Institute of Semiconductors, Chinese Academy of Sciences, Beijing 100083, China
}

\begin{abstract}
GaN films grown on sapphire by MOCVD were doped with $\mathrm{Mn}$ and $\mathrm{Cr}$ by solid state diffusion and characterized by various methods. Hall measurement shows that the samples still remain n-type after the diffusion. Secondary Ion Mass Spectroscopy (SIMS) results show a good diffusion of $\mathrm{Mn}$ and $\mathrm{Cr}$ inside GaN. X-ray diffraction (XRD) reveals no secondary phases in the samples. Superconducting quantum interference device (SQUID) results show that the samples are ferromagnetic up to room temperature. The possible origin of ferromagnetism is discussed.
\end{abstract}

\section{INTRODUCTION}

GaN has been applied in conventional optical and electron charge based devices. In electron spin based devices [1], GaN will continue to play a key role since GaN samples doped with transitional metals like Mn or $\mathrm{Cr}$ were predicted to be ferromagnetic with a high Curie temperature [2,3]. Though much work [2-7] has been done about $\mathrm{Mn}$ and $\mathrm{Cr}$ doped $\mathrm{GaN}$, many important issues like the origin of ferromagnetism, the change of c-lattice constant etc. remain open. In this paper, MOCVD grown GaN were doped with Mn and $\mathrm{Cr}$ by thermal diffusion. The doped samples were characterized and the origin of ferromagnetism was briefly discussed.

\section{EXPERIMENTS}

The unintentionally doped GaN wafers were grown at the same condition of $1100^{\circ} \mathrm{C}$ by MOCVD on sapphire (0001) substrates. Mn (100 nm) and Cr (51 $\mathrm{nm})$ thin films were deposited on the wafers by standard thermal evaporation under the vacuum of $10^{-6}$ torr. The coated wafers were then sealed in separate quartz tubes under the vacuum of $10^{-3}$ torr and annealed at $500{ }^{\circ} \mathrm{C}$ for 6 hours in a furnace. After annealing, the wafers were washed by $\mathrm{HCl}$ solution of $2 \mathrm{~mol} / \mathrm{L}$. The Mn doped wafer was labeled sample 1 and the $\mathrm{Cr}$ doped was labeled sample 2. The samples were characterized by X-ray fluorescence (XRF), SIMS, XRD, Hall measurement and SQUID.

\section{RESULTS AND DISCUSSION}

XRF results show that the $\mathrm{Mn}$ concentration is $0.02 \%$ for sample 1 and the $\mathrm{Cr}$ concentration is $2.68 \%$ for sample 2. SIMS results demonstrate that both $\mathrm{Mn}$ and $\mathrm{Cr}$ diffuse deep into $\mathrm{GaN}$ layer and their concentrations decrease gradually with increasing depth without accumulation inside GaN layer.

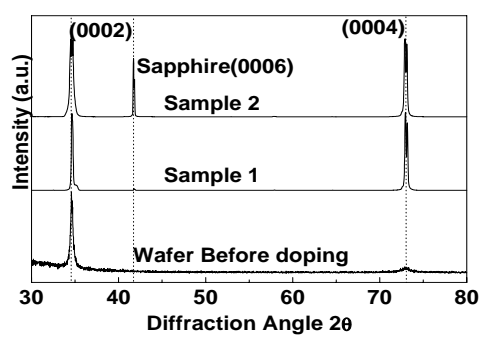

FIGURE 1. XRD $\theta-2 \theta$ curves for the two samples and the wafer before doping.

Fig. 1 shows the XRD results for the two samples and the wafer before doping. It can be observed that only the peaks related to h-GaN and sapphire appear. 
This indicates that no secondary phase or phase separation is detected. This result agrees with other reported results [5]. In addition, there is no obvious change in the peak positions after doping, which implies that doping causes no obvious change in clattice constant and this also agrees with the results in the literature [8].

Fig. 2 shows the magnetization as a function of applied field measured at $5 \mathrm{~K}$ and $300 \mathrm{~K}$. The magnetic field was applied parallel to the sample surface. The data shown in Fig.2 are the values extracted from the raw data by deducting the diamagnetic contribution of the substrate. Therefore, those data are more qualitative than quantitative. The curves in Fig. 2 are typical for a ferromagnetic material, indicating that the Curie temperature is around or over $300 \mathrm{~K}$. The saturation magnetization decreases as the temperature increases to $300 \mathrm{~K}$. The obtained Curie temperature is within the range of the values reported in the literature which vary from $10-25 \mathrm{~K}$ [6] to $940 \mathrm{~K}$ [7].
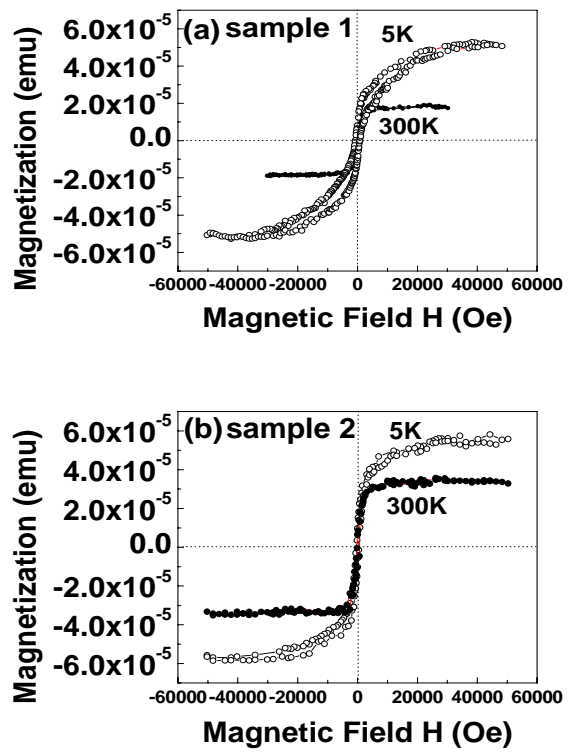

FIGURE 2. The magnetization versus magnetic field curves for sample 1 (a) and sample 2 (b) at $5 \mathrm{~K}$ and $300 \mathrm{~K}$.

Since SIMS and XRD results do not show any phase separation, it is possible that the observed ferromagnetism originates from an alloy of $\mathrm{GaN}$ and magnetic elements ( $\mathrm{Mn}$ and $\mathrm{Cr}$ respectively). Hall measurement shows that the samples are still n-type after doping. Other groups also reported that n-type GaN:Mn [6,7,9] and GaN:Cr [4] show ferromagnetism. Obviously these results can not be explained by the theory based on hole mediation [2]. The ferromagnetism in n-type $\mathrm{GaN}: \mathrm{Mn}$ and $\mathrm{GaN}: \mathrm{Cr}$ is possibly induced by the s-d exchange interaction, i.e. interaction of electron spins and localized moments of the transition metal, as previously proposed [10].

\section{CONCLUSION}

GaN doped with Mn and $\mathrm{Cr}$ by solid state diffusion shows room temperature ferromagnetism which comes from an alloy of $\mathrm{GaN}$ and magnetic elements (Mn, Cr). The observed ferromagnetic properties are possibly due to the s-d exchange interaction.

\section{ACKNOWLEDGEMENTS}

We are grateful to Dr. Y.G. Cao and Prof. Ning for XRD measurements. X.X. Zhang would like to thank the support by HK RGC.

\section{REFERENCES}

1. S. A. Wolf, D. D. Awschalom, R. A. Buhrman, J. M. Daughton, S. von Molnár, M. L. Roukes, A. Y. Chtchelkanova, and D. M. Treger, Science 294, 14881495 (2001).

2. T. Dietl, H. Ohno, F. Matsukura, J. Cibert, and D. Ferrand, Science 287, 1019-1022 (2000).

3. H. K.-Yoshida and K. Sato, Physica B 327, 337-343 (2003).

4. S. E. Park, H.-J. Lee, Y. C. Cho, S.-Y. Jeong, C. R. Cho, S. Cho, Appl. Phys. Letters 80, 4187-4189 (2002).

5. M. L. Reed, N. A. El-Masry, H. H. Stadelmaier, M. K. Ritums, M. J. Reed, C. A. Parker, J. C. Roberts, and S. M. Bedair, Appl. Phys. Letters 79, 3473-3475 (2001).

6. M. E. Overberg, C. R. Abernathy, S. J. Pearton, N. A. Theodoropoulou, K. T. McCarthy, and A. F. Hebard, Appl. Phys. Letters 79, 1312-1314 (2001).

7. S. Sonoda, S. Shimizu, T. Sasaki, Y. Yamamoto and H. Hori, J. Crystal Growth 237-239, 1358-1362 (2002).

8. M. B. Haider, C. Constantin, H. Al-Brithen, H. Yang, E. Trifan, D. Ingram, A. R. Smith, C. V. Kelly, and Y. Ijiri, J. Appl. Phys. 93, 5274-5281 (2003).

9.J. M. Baik, Y. Shon, T. W. Kang, and J.-L. Lee, Appl. Phys. Letters 84, 1120-1122 (2004).

10.K.-S. Huh, M.-C. Jeong, M.-H. Ham, J.-M. Myoung, W.Y. Lee, J.-M. Lee and S.-H. Han, Solid State Comm. 128, 119-123(2003). 
Copyright of AIP Conference Proceedings is the property of American Institute of Physics. The copyright in an individual article may be maintained by the author in certain cases. Content may not be copied or emailed to multiple sites or posted to a listserv without the copyright holder's express written permission. However, users may print, download, or email articles for individual use. 